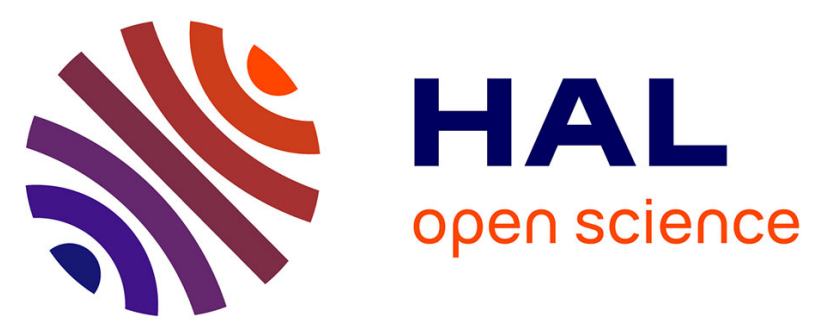

\title{
Improving LF Reader Antenna volume of detection for RFID token tag thanks to Identical Coaxial Loops (ICLs) and in/out-of phase multiple-loops structures
} Antoine Diet, Marjorie Grzeskowiak, Yann Le Bihan, Christophe Conessa

\section{- To cite this version:}

Antoine Diet, Marjorie Grzeskowiak, Yann Le Bihan, Christophe Conessa. Improving LF Reader Antenna volume of detection for RFID token tag thanks to Identical Coaxial Loops (ICLs) and in/out-of phase multiple-loops structures. IEEE RFID-TA 2014, Sep 2014, Tampere, Finland. 10.1109/RFIDTA.2014.6934229 . hal-01104665

\section{HAL Id: hal-01104665}

https://hal-centralesupelec.archives-ouvertes.fr/hal-01104665

Submitted on 20 Jan 2015

HAL is a multi-disciplinary open access archive for the deposit and dissemination of scientific research documents, whether they are published or not. The documents may come from teaching and research institutions in France or abroad, or from public or private research centers.
L'archive ouverte pluridisciplinaire HAL, est destinée au dépôt et à la diffusion de documents scientifiques de niveau recherche, publiés ou non, émanant des établissements d'enseignement et de recherche français ou étrangers, des laboratoires publics ou privés. 


\section{Improving LF Reader Antenna volume of detection for RFID token tag thanks to Identical Coaxial Loops (ICLs) and in/out-of phase multiple-loops structures}

\author{
Antoine DIET \\ L2S/DRE UMR 8506 - LGEP UMR 8507 \\ Plateau du Moulon, F-91192 Gif Sur Yvette, France \\ antoine.diet@u-psud.fr \\ Marjorie GRZESKOWIAK \\ Université Paris-Est, ESYCOM (EA 2552), \\ UPEM, ESIEE-Paris, CNAM, \\ F-77454 Marne-la-Vallée, France \\ marjorie.grzeskowiak@u-pem.fr
}

\author{
Yann LE BIHAN \\ LGEP UMR 8507 \\ Plateau du Moulon, F-91192 Gif Sur Yvette, France \\ yann.le-bihan@u-psud.fr \\ Christophe CONESSA \\ L2S/DRE UMR 8506 \\ Plateau du Moulon, F-91192 Gif Sur Yvette, France \\ christophe.conessa@1ss.supelec.fr
}

\begin{abstract}
This work concerns the prototyping of a low cost PCB based multiple loops antenna for LF $125 \mathrm{kHz}$ RFID application. The multiple loops structure is considered for the context of small tags traceability, for example token tags such as circular HitagS (1-2 cm of diameter). The targeted volume is $20 \times 20 \times 10 \mathrm{~cm}^{3}$ for the moment. The position of the tag can be parallel or orthogonal to the reader antenna plane, with a variation of the angle position, and is moving inside the volume of detection (small objects in a storage basket in practice). The dedicated conception of a reader antenna is necessary because the application targets a volume of detection rather than maximum range detection. Additionally, the position of the tag is not known. The design considerations are based on a combination of Identical Coaxial Loops (ICL) and in/out-of phase loops structures whose advantages are discussed in terms of mutual inductance in the simulation part. An optimum diameter of $7,5 \mathrm{~cm}$ is noticed for square loops case. The realization of this structure shows qualitatively good results for our objectives and emphasizes a tradeoff between the two effects of coupling due to the two structures combined. As the tag is moving inside the volume, with an orientation (tag position test) unknown, our observations drive to the conclusion that the detection will be effective. Some perspectives are also clearly identified to improve the structure in the future.
\end{abstract}

Keywords - RFID, LF, mutual inductance, antenna loops

\section{CONTEXT}

RFID is a mature technology with different possible mode of realization [1][2][3]. We emphasize here on LF magnetic coupling (125 kHz) RFID for small passive tags such as "token tags" or "glass-tag" whose detection is more critical than classical card tags. Our objective is to target detection "inside a volume" (called volume of control) of multiple small objects, in the range of centimeters, identified by a tag whose size is not exceeding $2 \mathrm{~cm}$ (of diameter). That is why token tags and glass-tags are considered for the tests. As the tag is composed by the RFID chip and its antenna, this size constraint is concerning the antenna design. To perform a sufficient volume of detection, the RFID reader antenna has to be widened, but this penalizes the magnetic coupling possibility with the size constrained tag antennas. We fixed a first limit of $10 \mathrm{~cm}$ for the height of the volume of control (over a surface as wide as possible), corresponding to a possible manipulation of tagged object in a storage basket.

A structure optimization is mandatory for the reader antenna in order to improve the performance of the detection system [4][5], quantified here by the volume of detection and parameterized by the tag geometrical position inside this volume (parallel or orthogonal to the reader antenna plane). To increase the mutual inductance, $M$, the geometry of the reader antenna is focusing multiple loops structure.
Case 1
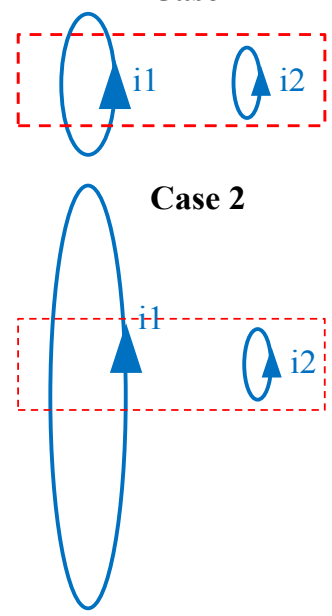

Case 3

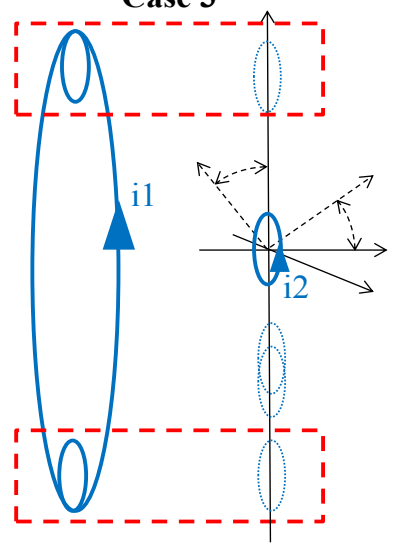

Figure 1: Illustration of multi-loops advantage for the maximization of antennas inductive mutual coupling (delimited area). The position of the tag can be changed and creates different areas

As seen in figure 1, the presence of multiple loops in the reader antenna structure creates some geometrical similarities that can increase locally the coupling effect. If we consider the 
case 3 and identify $\Gamma_{1}$ and $\Gamma_{2}$ as the respective circulations of the reader multiple loop antenna (driven by a current $I_{l}$ ) and the tag loop antenna (driven by a current $I_{2}$ ), the Neumann formulae is expressed by (1) where $r_{12}$ is the distance from $d l_{1}$ to $d l_{2}$.

$$
M=\frac{\mu_{0}}{4 \pi} \oint_{\Gamma_{l}} \oint_{\Gamma_{2}} \frac{\overrightarrow{d l_{1}} \cdot \overrightarrow{d l_{2}}}{r_{12}}
$$

The reader multiple loop antenna of case 3 presents a local loop that we can delimit as circulation $\Gamma_{l a}$ and the rest of the antenna loop can be identified by its circulation $\Gamma_{l b}$. Thus, the mutual inductance $M$ can be expressed as the sum of $M_{a}$ and $M_{b}$ as justified by (2)

$$
\begin{aligned}
M & =\frac{\mu_{0}}{4 \pi} \oint_{\Gamma_{1}=\Gamma_{1}^{a}+\Gamma_{1}^{b}} \oint_{\Gamma_{2}} \frac{\overrightarrow{d l_{1}} \cdot \overrightarrow{d l_{2}}}{r_{12}} \\
& =\frac{\mu_{0}}{4 \pi}\left[\oint_{\Gamma_{I}^{a}} \oint_{\Gamma_{2}} \frac{\overrightarrow{d l_{1}} \cdot \overrightarrow{d l_{2}}}{r_{12}}+\oint_{\Gamma_{1}^{b}} \oint_{\Gamma_{2}} \frac{\overrightarrow{d l_{1}} \cdot \overrightarrow{d l_{2}}}{r_{12}}\right] \\
& =M_{a}+M_{b}
\end{aligned}
$$

Equation (2) is the starting point of the reader antenna structure proposed, where the value of $M$ is maximized over the volume of detection by creating local geometrical similarities. The multiple loops structure can create some local loop with consequently mutual inductance $M_{a}$ much higher than $M_{b}$ depending on the tag antenna loop, its position and distance. To increase the magnetic flux inside the volume, the ICL structure is introduced. However, the tag position is a determining parameter which creates some critical cases of zero-coupling, for example if the tag is orthogonal to the reader antenna plane, even in an ICL structure [6][7][8][9]. To overcome this, an alternation of in-phase and out-of-phase loops creates the needed orthogonal magnetic flux that can efficiently be integrated by the effective surface of the tag loop antenna.

We present in the following parts the theoretical and empirical analysis with MATLAB and the test with the realization of the two levels multiple loops structure where the ICL are alternately inversed in order to create the wanted inphase and out-of-phase excitation currents of the loops.

\section{MUTUAL INDUCTANCE CONSIDERATIONS}

\section{A. Theoretical results}

As known in LF magnetism theory, the mutual inductance, $M_{12}$, is the flux of the magnetic field created by a primary magnetic antenna integrated on the equivalent surface of the second antenna [10][11]. This effect is reciprocal, thus $M_{12}=$ $M_{21}=M$. The magnetic field $\left(B_{r}, B_{\theta}, B_{y}\right)$ created at an arbitrary point $(x, y, z)$ by a circular loop of radius $\mathrm{R}$, centered in $(0,0$, $0)$ in the plane $(x O z)$ and driven by a current $\mathrm{I}$ is expressed, in cylindrical coordinates by (3), where $r$ is the distance between the loop center and the point $(x, y, z)$ and the functions $E()$ and $K()$ the complete elliptic integral of second and first kind respectively.

$$
\left\{\begin{array}{l}
B_{r}=\frac{I \mu_{0} y}{2 \pi\left[(R+r)^{2}+y^{2}\right]^{1 / 2} r}\left[\frac{R^{2}+r^{2}+y^{2}}{(R-r)^{2}+y^{2}} E(k)-K(k)\right] \\
B_{\theta}=0 \\
B_{y}=\frac{I \mu_{0}}{2 \pi\left[(R+r)^{2}+y^{2}\right]^{1 / 2} r}\left[\frac{R^{2}-r^{2}-y^{2}}{(R-r)^{2}+y^{2}} E(k)-K(k)\right] \\
k^{2}=\frac{4 R r}{(R+r)^{2}+y^{2}}
\end{array}\right\}
$$

Equation (3) is processed under MATLAB with spatial discretization, and will be used in the simulation part.

By defining a secondary loop in a coaxial and parallel plane case, we can analytically compute $M$, as expressed by (4). $R_{I}$ and $R_{2}$ are the radii of primary and secondary loops respectively, with a distance $d$ between the two centers (thus equivalent to $y$ in our case).

$$
\begin{aligned}
M & =\frac{\mu_{0}}{4 \pi} \oint_{\Gamma_{1}} \oint_{\Gamma_{2}} \frac{\overrightarrow{d l_{1}} \cdot \overrightarrow{d l_{2}}}{r_{12}} \\
& =-\mu_{0} \sqrt{R_{1} R_{2}}\left[\left(\rho-\frac{2}{\rho}\right) K(\rho)+\frac{2}{\rho} E(\rho)\right] \\
& =\mu_{0} \sqrt{R_{1} R_{2}} X(\rho) \\
\rho & =\frac{2 \sqrt{R_{1} R_{2}}}{\sqrt{\left(R_{1}+R_{2}\right)^{2}+d^{2}}} \leq 1
\end{aligned}
$$

If we want to analyze equation (4) in the case of the multiple loops structure, as equation (2) expressed, we had to make some hypothesis for the definition domain of $\rho$ before comparing $M_{a}$ and $M_{b}$.

We suppose that the two coaxial and collinear loops considered are at a minimum distance equal to the minimum of the two radii, that is to say: $d>\min \left(R_{l}, R_{2}\right)$. The maximum value for $\rho$ is consequently expressed by (5), if we use $R_{1}=$ $n R_{2}$.

$$
\left.\begin{array}{c}
d \geq \min \left(R_{1} ; R_{2}\right) \\
R_{1}=n R_{2} \\
\rho^{2}=\frac{2 R_{1} R_{2}}{\left(R_{1}+R_{2}\right)^{2}+d^{2}} \leq \frac{2 n R_{2}^{2}}{\left(n R_{2}+R_{2}\right)^{2}+R_{2}^{2}} \\
\rho^{2} \leq \frac{2 n}{(n+1)^{2}+1} \\
\rho_{\text {max }}=\sqrt{\frac{2 \sqrt{2}}{2 \sqrt{2}+4}}(\approx 0.6436)
\end{array}\right\}
$$

Consequently, we can plot the value of $X(\rho)$ defined in (4) with respect to the values of $\rho$ between 0 and $\rho_{\max }$ for the interpretation. 


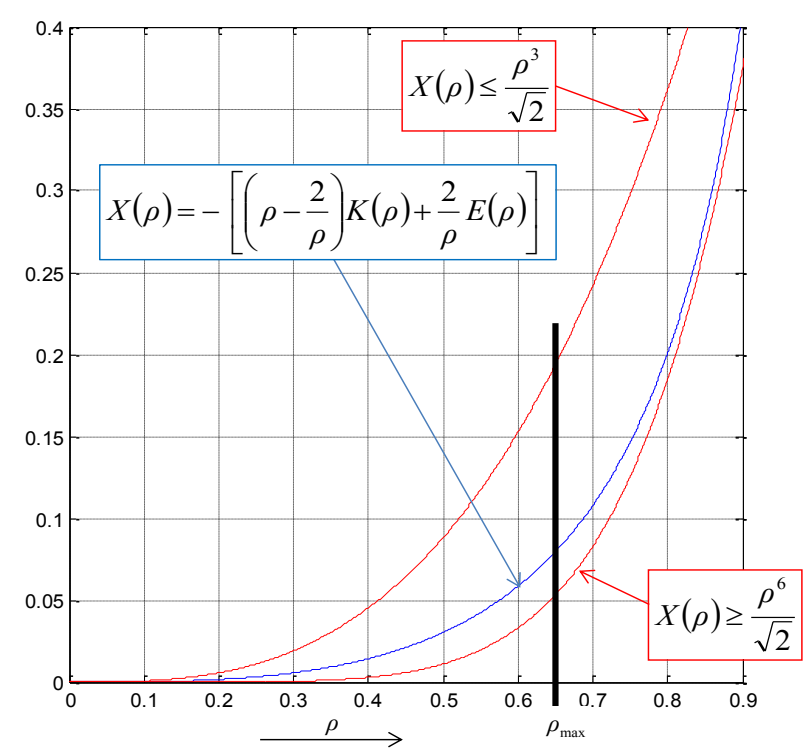

Figure 2: $X(\rho)$ in function of $\rho$.

As can be seen in figure 2, X( $\rho)$ can be empirically minimized by the function $\sqrt{2} \rho^{3}$ and maximized by the function $\sqrt{ } 2 \rho^{6}$. This will be useful for comparison of $M_{a}$ and $M_{b}$ which are the case where $M$ is considered for two part of the reader antenna loop, "a" and "b", while a tag antenna of radius $\mathrm{R}_{2}$ is present at a distance $d$ (the hypothesis is still coaxial and collinear in this analytical part). We can find a relationship between $M_{a}$ and $M_{b}$ thanks to equations (4) and figure 2 results, by introducing $R_{l a}=x R_{l b}$ and centering at $(0$, $0,0)$ the loops in figure 1 , case 3 . This drives to equations (6), (7) and (8).

$$
\begin{aligned}
R_{1 b} & =x R_{1 a} \\
M_{a} & =M\left(\rho_{a}\right)=\mu_{0} \sqrt{R_{1} R_{2}} X\left(\rho_{a}\right) \\
M_{b} & =M\left(\rho_{b}\right)=\mu_{0} \sqrt{R_{1} R_{2}} X\left(\rho_{b}\right)
\end{aligned}
$$

From (6) we can evaluate a relation between $\rho_{a}$ and $\rho_{b}$.

$$
\begin{aligned}
& \frac{\rho_{a}}{\rho_{b}}=\frac{2 \sqrt{R_{1 a} R_{2}}}{2 \sqrt{R_{1 b} R_{2}}} \frac{\sqrt{\left(R_{1 b}+R_{2}\right)^{2}+d^{2}}}{\sqrt{\left(R_{1 a}+R_{2}\right)^{2}+d^{2}}}=\frac{1}{\sqrt{x}} \frac{\sqrt{\left(x R_{1 a}+R_{2}\right)^{2}+d^{2}}}{\sqrt{\left(R_{1 a}+R_{2}\right)^{2}+d^{2}}} \\
& \frac{\rho_{a}}{\rho_{b}}=\sqrt{\frac{\left(x R_{1 a}+R_{2}\right)^{2}+d^{2}}{x\left(R_{1 a}+R_{2}\right)^{2}+x d^{2}}}=\sqrt{\frac{x^{2} R_{1 a}{ }^{2}+x 2 R_{1 a} R_{2}+R_{2}{ }^{2}+d^{2}}{x\left[\left(R_{1 a}+R_{2}\right)^{2}+d^{2}\right]}} \\
& \rho_{a} \approx \sqrt{x} \rho_{b} \quad\left(x R_{1 a}>>R_{1 a}, R_{2}, d\right)
\end{aligned}
$$

Thus, the mutual inductance $M_{a}$ and $M_{b}$ can be compared thanks to $X\left(\rho_{a}\right)$ and $X\left(\rho_{b}\right)$ if we consider $X\left(\rho_{a}\right)$ not equal to 0 and use the empirical function in figure 2 , see (8). The result of equation (8) enables to consider that the mutual inductance is locally impacted by a factor which can be evaluated between $\mathrm{x}^{-1}$ and $\mathrm{x}^{-5 / 2}$ if the radius of the wider loop is $\mathrm{x}$ times the radius of the smaller one in a multiple loop structure. As a consequence, the mutual inductance is highly improved locally by these small loops. However, the distance is another penalty to overcome because the reduction of the antennas sizes for the tag antenna and the local small loop implies a lowering of the maximum range. As our applications is targeting a volume of detection rather than a maximum distance, the use of many small ICLs is considered for increasing the magnetic flux.

$$
\begin{aligned}
\frac{\rho_{b}^{3}}{\sqrt{2}} \frac{\sqrt{2}}{\rho_{a}^{3}} & \geq \frac{X\left(\rho_{b}\right)}{X\left(\rho_{a}\right)} \geq \frac{\rho_{b}^{6}}{\sqrt{2}} \frac{\sqrt{2}}{\rho_{a}^{6}} \\
X\left(\rho_{a}\right) \frac{1}{x \sqrt{x}} & \geq X\left(\rho_{b}\right) \geq X\left(\rho_{a}\right) \frac{1}{x^{3}} \\
\frac{M_{a}}{x} & \geq M_{b} \geq \frac{M_{a}}{x^{2} \sqrt{x}}
\end{aligned}
$$

Evaluating the mutual inductance in such more complex structure will now be done by simulation with MATLAB. Also, the position and orientation of the antenna can be varied for different distance.

\section{B. Simulations}

The mutual inductance is difficult to evaluate analytically for non-coaxial and non-collinear cases. With some calculator, it is possible to evaluate it numerically by discretizing equation (3) and defining a surface to evaluate the magnetic flux integration. As the mutual inductance is reciprocal, we fix the primary circular loop generating the magnetic field of equation (3) to be the tag antenna with a diameter of $1 \mathrm{~cm}$. The reader antenna is consequently represented by the surface of integration that we can define arbitrarily in the space. A square surface is preferred for simplicity of calculus and future realization (following part). A current of $1 \mathrm{~A}$ is chosen in order to evaluate directly the value of $M$ by a sum of the orthogonal component of the magnetic field over the surface delimited for the reader antenna. The space discretization is $500 \mu \mathrm{m}$.

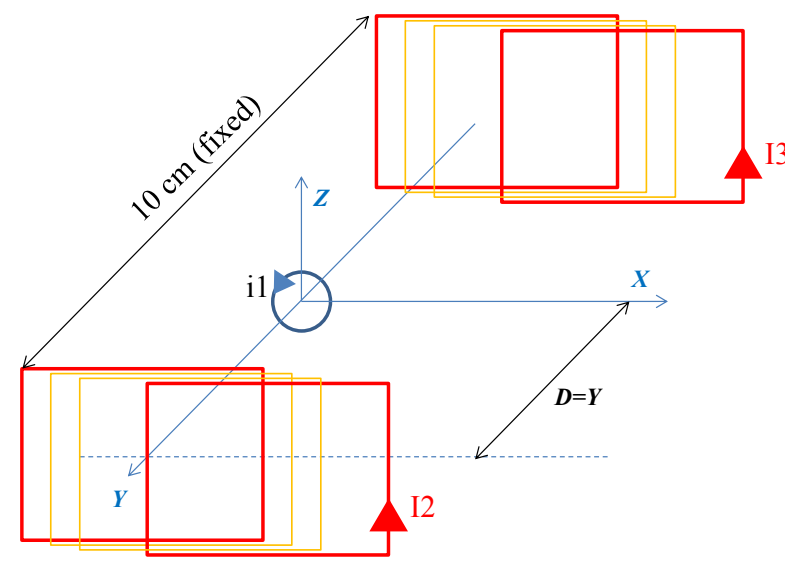

Figure 3 : Configuration of the primary loop antenna and secondary square antenna, in ICL case, for the simulation of M (MATLAB)

Figure 3 summarizes the first configuration considered for the evaluation of $M$. When the antennas are coaxial, and in parallel planes, at a fixed distance, the mutual inductance in function of the square antenna is positive. $M$ is 0 when the length of the square antenna is 0 or when it reaches an infinite value. It exists a maximum for $M$ in function of this length, which varies with the distance $d$ due to the magnetic field 
distribution created by the primary loop (of constant diameter). The ICL structure presents a fixed distance of $10 \mathrm{~cm}$ between the two square loops. As the current $I 2$ and $I 3$ are in the same orientation, the value of $M$ in such case is evaluated by the sum of the first loop $M_{\text {first }}$ value at a distance $d$ with the $M_{\text {second }}$ value at a distance $d^{\prime}=10 \mathrm{~cm}-d$, as reported in figure 4 .

Figure 4 presents the results of the simulation for the configuration of figure 3 in the case of a single square antenna (top) and in the case of the ICL structure (bottom). It can be seen in Figure 4 that the ICL structure is highly improving the ability to power and detect the tag by magnetic coupling in our delimited volume, as the worst case is for a distance of $5 \mathrm{~cm}$. The optimal length of the reader antenna is dependent on the distance $d$ and increases with the size of this square surface.

In our case where the height of the volume of detection implies a distance of $10 \mathrm{~cm}$ for the ICL, a tradeoff can be evaluated around 7 to $8 \mathrm{~cm}$ for the average length of the reader antenna square loop, driving to a mutual inductance value of more than 50 pico-Henry, for a single turn inductance case.
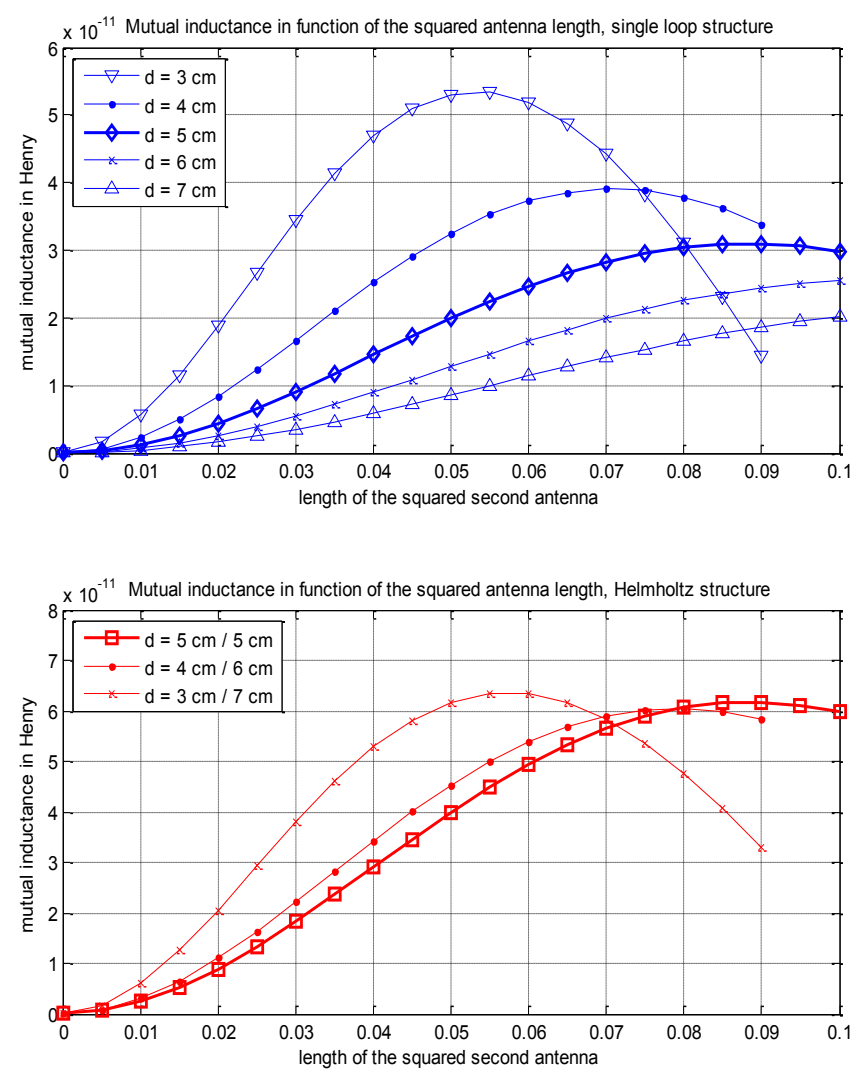

Figure4: Results of $M$, at different distance in a parallel plane case, in function of the length of the square reader antenna, in meter. Case of a single reader antenna (top) and ICL (bottom), as exposed in figure 3.

Figure 3 and figure 4 illustrate the case where the tag antenna is in a parallel plane. In an application of traceability of small object tagged, the position can be randomized. Consequently, the ICL structure has to be improved in the idea to detect some configuration where the tag is not parallel. A worst case is the orthogonal position, as seen in figure 5 .
Induced currents $\mathbf{I 2}$ and $I 3$ are supposed inverted

(out-of-phase case)

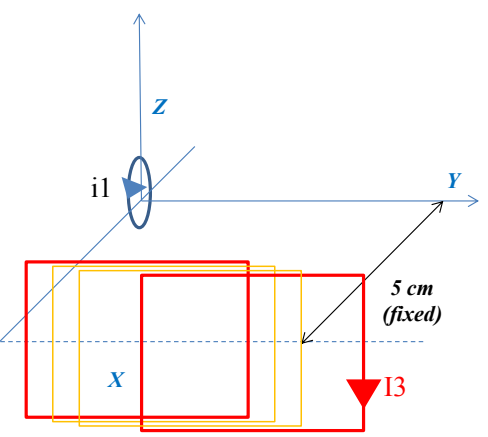

Figure 5: Configuration of the primary loop antenna and secondary square antenna, for orthogonal positioning of the tag with in-phase / outof-phase loops, for the simulation of M (MATLAB)

The orthogonal position represented in Figure 5 can be detected with the combination of in-phase and out-of-phase loops [12], as the direction of the current I2 and I3 illustrate this. This ability is due to the orientation of the magnetic flux integrated on these surfaces, which value is of the same sign due to the difference of phase $\left(180^{\circ}\right)$ of the two harmonic current. To realize this, it just needs to invert the turning rotation of the loops.

The simulation of $M$ is done with the discretization of equation (3) and by setting the distance on $x$-axis at $5 \mathrm{~cm}$. The integration surface is supposed to be a square, parallel to the $y 0 z$ plane, with a length of $7,5 \mathrm{~cm}$, and the position of the secondary (reader) loop center is moved from 0 to $10 \mathrm{~cm}(\mathrm{y}-$ axis). With the hypothesis of a second out-of phase loop (coplanar configuration) whose center is $10 \mathrm{~cm}$ shifted from the first loop one (y-axis), it is possible to evaluate the total mutual inductance $M$, as seen in figure 6 .

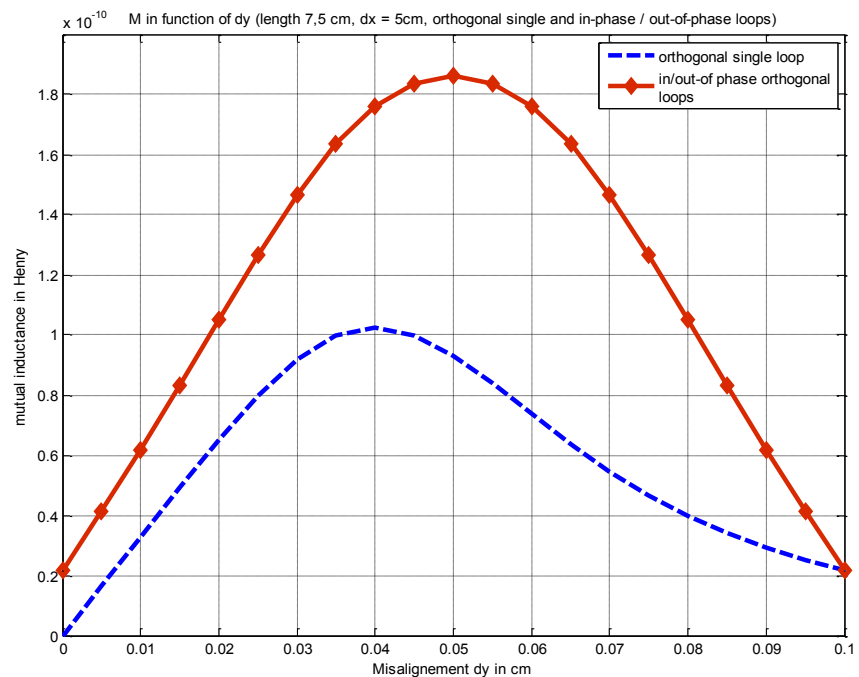

Figure 6: $M$ at different misalignment dy from 0 to $10 \mathrm{~cm}$, in an orthogonal configuration at $\mathrm{dx}=5 \mathrm{~cm}$, for a length of the square reader antenna of $7,5 \mathrm{~cm}$. Cases of a single reader loop antenna and two in/out-of phase loops, which centers are spaced by $10 \mathrm{~cm}$, as exposed in figure 5 .

Figure 6 confirms that it is possible to improve the detection of a tag in orthogonal position by using the in/out-of phase loops configuration. As this requires the loops to be in 
the same plane, this is compatible with a ICL structure. Consequently, several different positions of the tag can be detected by this combination. Moreover, there exists a destructive interaction between the ICL structure and the in/out-of phase loops structure because the magnetic field increases in orthogonal directions by these two improvements. A tradeoff should consequently be made in order not to reduce the advantages of one structure compared to the other one. In practice, this means that the distance from the loop centers should be in the same range and could be optimized in the future.

The following part will present the prototype based on these theoretical and simulation results. Figure 3 and figure 5 were investigated in the idea to combine the ICLs and the in/out-of phase loops structures.

\section{TESTS OF THE PROTOTYPE}

The RFID reader socket board is the RFID-UNI-EVAL kit from Ib technology with its $125 \mathrm{kHz}$ module. The hitagS token tag are used for the detection test in parallel (or horizontal) and orthogonal position. As seen in figure 7, The RFID reader uses an external antenna that can be changed with our design. This antenna should present an inductive impedance of $700 \mu \mathrm{H}$ at $125 \mathrm{kHz}$ and a quality factor of no more than 20 (classical in RFID application for the necessary bandwidth) [13].

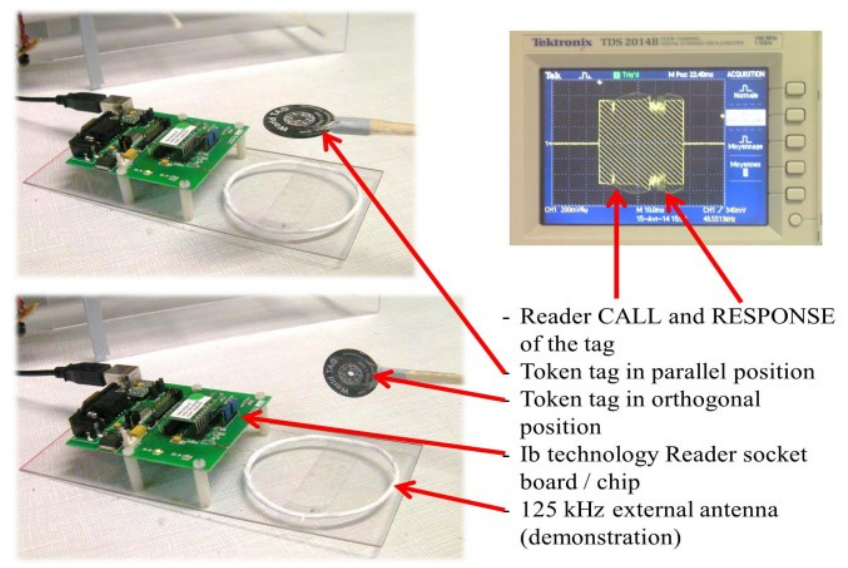

Figure 7: Ib technology RFID board (125 kHz module). Tests with hitagS token tag in the two configurations (demonstration antenna). Illustration of the load modulation (oscilloscope in envelope detection mode)

As discussed in the previous part, our structure would combine ideally the ICL structure and the in/out-of phase loops structure. We decide to realize a serialization, tuned with a series capacitor, of four ICLs, realized on PCB (25 turns on each side, average length of 7,5 cm, $250 \mu \mathrm{H}$ each loop of an ICL) and to invert alternately the rotation direction of the loops, as is illustrated with strokes in figure 8 .

The token tag is moved inside the volume of control delimited by the PCB loops and also outside it. We can summarize the detection performances by these observations:

- Inside the volume of control, and in each volume determined by the ICL, a tag in parallel position is detected, as in [14]. If a tag in this position is moving inside the volume, it will be detected, except if its trajectory is exactly following the symmetry axis of the structure ("between" all the loops). An angle variation of $45^{\circ}$ is possible.

- Inside the volume of control, a tag in orthogonal position is detected in the vicinity of the copper line of the PCB, as seen in figure 9. The delimitation of the detection area is following a thick line parallel to the symmetry axes of the structure. An angle of $30^{\circ}$ is possible but reduces the area of detection.

- Outside the volume, a maximum range of $3 \mathrm{~cm}$ is possible in the parallel position for the tag, except if the tag is facing the space between two loops.

- Outside the volume of detection, the orthogonal position is well detected at $4 \mathrm{~cm}$ and for an angle tolerance of more than $40^{\circ}$. The effect of in/out-of phase structure is stronger.
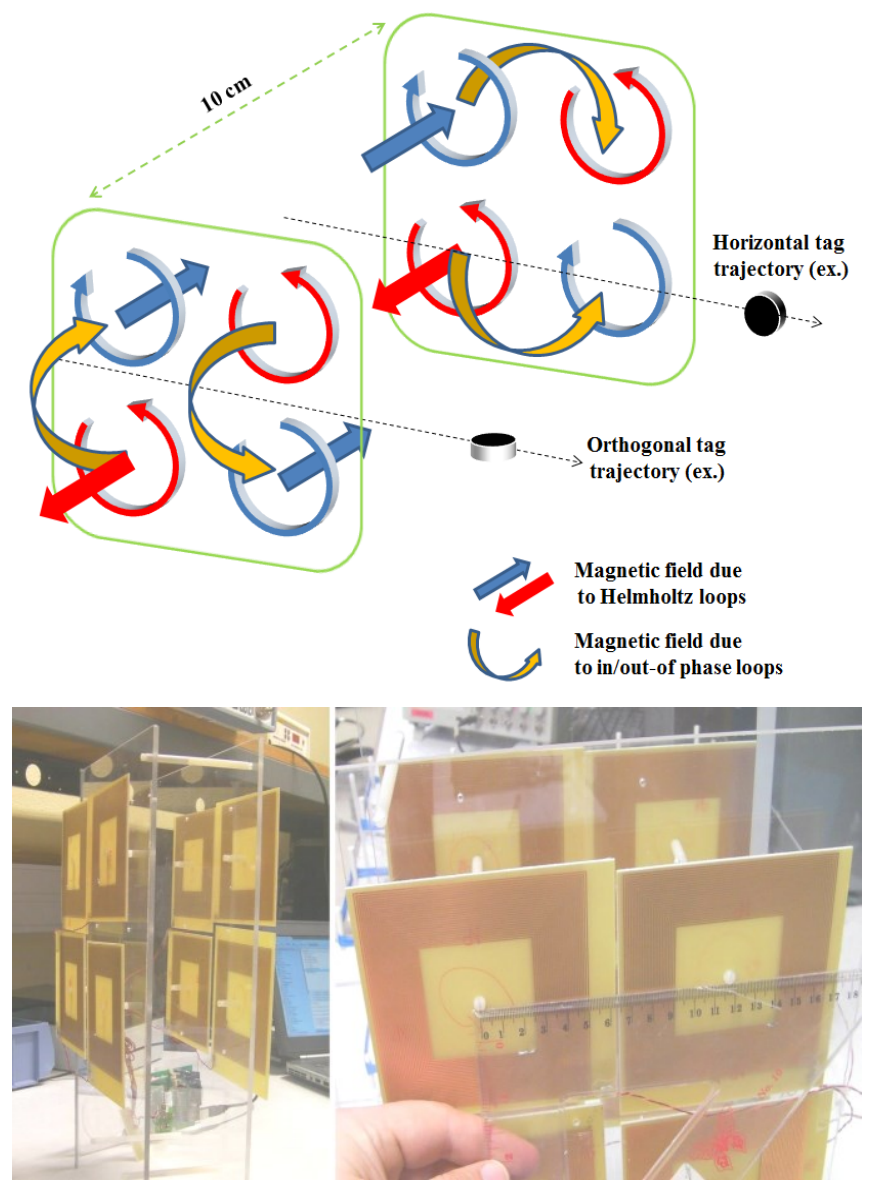

Figure 8: Structure of the prototype and its realization with PCB loops. The structure enables the different magnetic flux illustrated in the schematic (top), where the "tests trajectories" of the tags are added.

The observation drives us to the conclusion that the ICLs effect are stronger than the in/out-of loops effect inside the volume of detection, and this ratio is inversed outside the volume. The ability to detect a tag around the volume, including inside and an area outside, is interesting because the two configurations are possible in multiple areas with an angle variation. This is highly positive for consideration of randomly positioned tags. 
To conclude, the prototype showed an efficient realization of multiple loops antenna structure, based on the considerations analyzed in simulations.

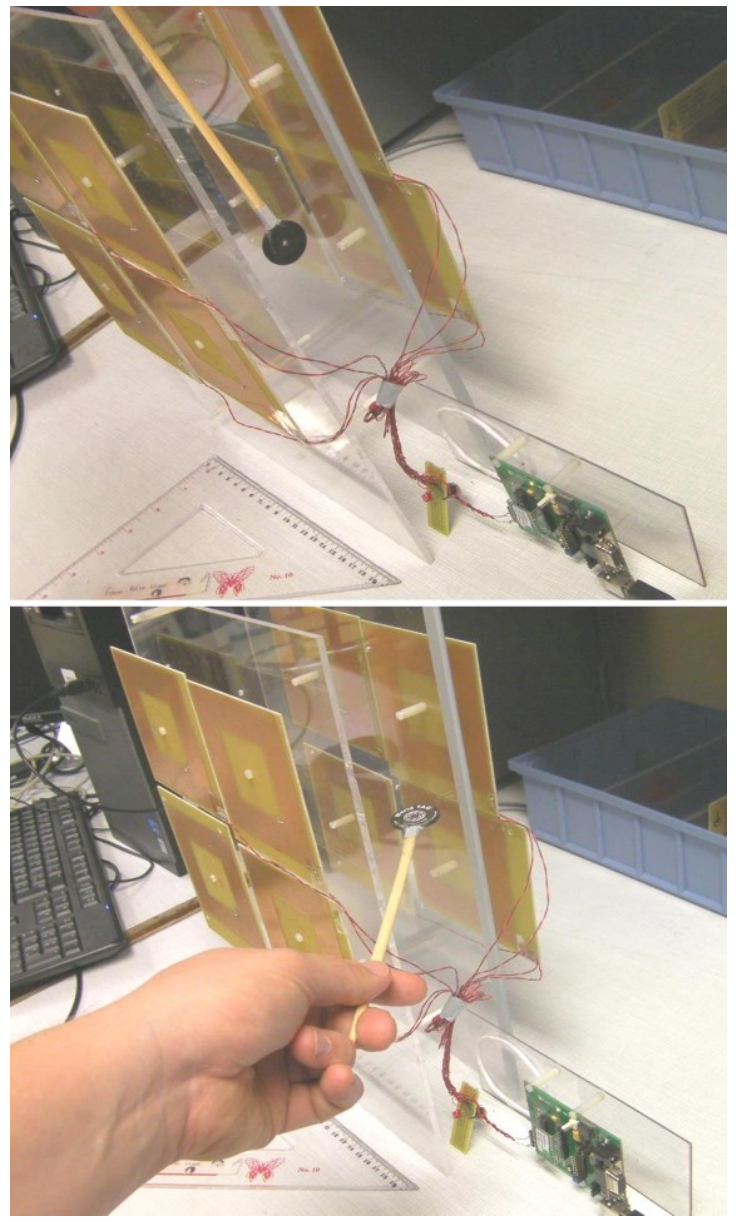

Figure 9: Tests of the tag detection in orthogonal configuration (tests are inside the volume of detection here).

\section{CONCLUSIONS}

This works has summarized our approach and consideration for the design of a multiple loop antenna, adapted in the context of small tagged object traceability. The optimization of mutual coupling when the loops sizes are disproportionate implies the analysis and design of complex structures based on multiple loops, whose analytic approach and simulation is difficult. As the multiple loops structure approach is theoretically justified thanks to equations in part I, the impact of ICLs and in/out-of loops is studied in simulations and completes our design consideration.

The prototype based on these results showed good and promising performance for the detection of small token hitagS RFID tags. The possible detection of the two position tag configurations is of great interest for the targeted application, where the position is not known. Our tests showed a tradeoff between the effects of the two loops structures that drives us to investigate an analysis of optimal placement of the PCB loops in the idea to optimize the magnetic field distribution, and control its orientation.

Alternately, multiple loops antenna solutions based on commutated loops are to be investigated and could provide an interesting switching mode of operation between these two structures.

\section{REFERENCES}

[1] D. Paret. Identification Radio-Fréquences et Cartes à puce sans contact. Dunod, Paris, 2000.

[2] K. Finkenzeller. RFID handbook, fundamentals and applications in contactless cards and identifications. $2^{\text {nd }}$ ed., Wiley and Sons, 2003.

[3] S. Barbu. Conception et réalisation d'un système de métrologie RF pour les systèmes d'identification sans contact à 13,56 MHz. Thèse de Doctorat, 2005-313, Université de Marne la Vallée, 2005.

[4] H. Hirayama et al. A New Scheme to Avoid Null Zone for HF Band RFID with Diversity Combining of Loop Antennas. IEICE transactions on Communications, Vol. 93, pp. 2666-2669, 2010.

[5] X Qing, Z. Chen. Proximity effects of Metallic Environments on HF RFID Reader Antenna: Study and Applications. IEEE transactions on Antennas and Propagation, Vol. 55, pp. 3105-3111, 2007.

[6] U. Azad, H. C. Jing, Y. E. Wang. Link budget and capacity performance of inductively coupled resonant loops. IEEE transactions on antennas and propagation, May 2012, pp 2453-2461.

[7] S. Kawdungta, C. Phongcharoenpanich, D.Torrungrueng. Design of a novel dual loop gate antenna for radio frequency identification (RFID) systems at low frequency band. Progress in Electromagnetics Research C, Vol. 12, 1-14, 2010.

[8] W. Aerts, E. de Mulder, B. Preneel, G. Vandenbosch, I. Verbauwhede. Dependence of RFID Reader Antenna Design on Read Out Distance. IEEE transactions on Antennas and Propagation, Vol. 56, $n^{\circ} 12$, December 2008

[9] M. Ahmad, A. Mohan. Multiple-bridge-loop RFID Reader Antenna for improved Positioning and localisation. Electronics Letters, Vol. 48, $n^{\circ} 16$, August 2012.

[10] C. Zierhofer, E. Hochmair. Geometric approach for coupling enhancement of magnetically coupled coils. IEEE transacions on Biomedical Engineering, Vol. 43, n7, pp. 708-714, July 1996.

[11] K. Fotopoulou, B. Flynn. Optimum antenna coil structure for inductive powering of passive RFID tags. Proc. IEEE Int. Conf. RFID, 2007, pp. 71-77.

[12] TI SCBA034. HF Antenna design notes. Technical application report, Texas Instruments, 11-08-26-003, ed. $3^{r d}$, September 2003.

[13] Microchip Applications Notes (AN) $n^{\circ} 680$, passive RFID basics, and $\mathrm{n}^{\circ} 710$, Antenna circuit design for RFID applications. Microchip technology Inc., 2003.

[14] K. Wang, A. Diet, S. Abou Chakra, C. Conessa, M. Grzeskowiak, T. Bouaziz, S. Protat, D. Delcroix, L. Rousseau, G. Lissorgues, A. Joisel. Detecting range and coupling coefficient tradeoff with a multiple loops reader antenna for small size RFID LF tags. IEEE International Conference on RFID-Technology and Applications 2012, IEEE RFIDTA, 2012 november 5-7, Palais de la Méditerranée, Nice, France 